\title{
Telomeres positively correlate with pace-of-life and elongate with age in a wild mammal
}

\author{
Mathilde Tissier ${ }^{1}$, Patrick Bergeron ${ }^{2}$, Dany Garant ${ }^{2}$, Sandrine Zahn ${ }^{3}$, Francois Criscuolo ${ }^{4}$, \\ and Denis Réale ${ }^{5}$ \\ ${ }^{1}$ Bishop's University \\ ${ }^{2}$ Université de Sherbrooke \\ ${ }^{3}$ Centre National de la Recherche Scientifique \\ ${ }^{4} \mathrm{CNRS}$ \\ ${ }^{5}$ Université du Québec à Montréal
}

October 14, 2021

\begin{abstract}
Understanding ageing and the diversity of life histories is a cornerstone in biology. Telomeres, the protecting caps of chromosomes, are thought to be involved in ageing, cancer risks and to modulate life-history strategies. They shorten with cell division and age in somatic tissues of most species, possibly limiting lifespan. The resource allocation trade-off hypothesis predicts that short telomeres have thus co-evolved with early reproduction, proactive behaviour and reduced lifespan, i.e. a fast Pace-of-Life Syndrome (POLS). Conversely, since short telomeres may also reduce the risks of cancer, the anti-cancer hypothesis advances that they should be associated with slow POLS. Conclusion on which hypothesis best supports the role of telomeres as mediators of life-history strategies is hampered by a lack of study on wild short-lived vertebrates, apart from birds. Using seven years of data on wild Eastern chipmunks Tamias striatus, we highlighted that telomeres elongate with age and do not limit lifespan in this species. Furthermore, short telomeres correlated with a slow POLS in a sex-specific way. Females with short telomeres had a delayed age at first breeding and a lower fecundity rate than females with long telomeres, whereas those differences were not recorded in males. Our findings support most predictions adapted from the anti-cancer hypothesis, but none of those made under the resource allocation trade-off hypothesis. Results are in line with an increasing body of evidence suggesting that resource allocation trade-offs alone cannot explaining the diversity of telomere length in adult somatic cells and life-histories observed across the tree of life.
\end{abstract}

\section{Hosted file}

MS-Mol-Ecol-Telomeres2021_VF.DOCX available at https://authorea.com/users/441095/articles/ 541679-telomeres-positively-correlate-with-pace-of-life-and-elongate-with-age-in-a-wildmammal 